\title{
Projeto digital storytelling: trabalhando narrativas digitais com idosos
}

Helena Brandão Viana*

\section{Resumo}

Este século marcou uma transição na forma de narrar histórias, já que é possível faze-lo hoje de forma mais envolvente através da incorporação de áudio, imagens, clipes e músicas produzidas em uma plataforma digital. O objetivo geral desta pesquisa é relatar a experiência na participação de um projeto de Narrativas Digitais com idosos no Canadá. Esse projeto aconteceu em 10 semanas, num formato de workshop, aonde o pesquisador ensinou o participante a elaborar e escrever uma estória e informou quais seriam os recursos utilizados nesse processo. Esse trabalho foi realizado com pessoas idosas, que foram convidadas a participar do workshop. Para esse projeto foi utilizado um software para gravação do áudio da voz do participante, colocação das imagens e montagem da sonoplastia. Os estudos no Canadá com Narrativas Digitais entre a população idosa, tem mostrado resultados positivos na interação social, estimulação cognitiva e bem-estar percebido.

Palavras-chave: Narrativas. Digitais. Idosos. Tecnologia.

\section{Introdução}

A proliferação de tecnologias digitais tem promovido novas formas de alfabetização, incluindo possibilidade de contar histórias utilizando recursos tecnológicos. A Tecnologia atualmente também nos permite a combinação de vários modos semióticos, incluindo verbal, visual, auditivo, espacial e gestual. Usando duas ou mais combinações simultaneamente, os autores de histórias digitais podem explorar múltiplas possibilidades e estratégias para apresentar suas estórias. Por exemplo, eles podem colocar uma foto durante a narração oral, música, uma legenda, efeitos visuais como zoom entre outros recursos e componentes visuais. Os narradores podem, portanto, experimentar uma infinidade de recursos semióticos para expressar significados e emoções diferentes (PRINS, 2016).

A narrativa digital (ND) é uma ferramenta relativamente recente para objetivos instrucionais e de pesquisa. $\mathrm{O}$ presente século traz uma transição na

* Doutora em Atividade Física Adaptada e Qualidade de Vida; Pesquisadora Visitante na Simon Fraser University No Canadá - Parceira Na Pesquisa Storytelling For Elderly; Professora no Mestrado Profissional em Educação no UNASP; Coordenadora do Grupo de Pesquisa Edetec, Educação e Tecnologia.

$\rightarrow$ http://dx.doi.org/10.5335/rbceh.v17i1.8010 
narrativa de fatos ou ideias, já que as pessoas começaram a contar estórias em ambientes digitais. Estórias mais envolventes e emocionantes podem ser desenvolvidas incorporando áudio, imagens, clipes e música em narrativas escritas em uma plataforma digital. A narrativa digital também pode ser usada para combinar aplicações tecnológicas com abordagens pedagógicas ou de pesquisa, sendo este um campo emergente. O termo "narrativa digital" (ND), também conhecido como "Digital Storytelling”(DST), foi cunhado por Dana Atchley, que projetou imagens, fotos de família e filmes em uma tela grande nos anos 80 (MCLELLAN, 2007). Na educação, o uso das narrativas digitais (ND), cresceu significativamente nas escolas, desde 0 jardim de infância até o nível universitário (KOCAMAN-KAROGLU, 2016).

Desde então, vários autores iniciaram processos e criaram diretrizes para a criação de ND (KOCAMAN-KAROGLU, 2016; KOTLUK, 2015; LEE et al., 2014; LETHERBY; DAVIDSON, 2015).

Coletar informações com a população idosa para fins de pesquisa através de relatos de estórias dos mesmos, não é um fato novo. Há relatos na literatura de pesquisas realizadas com idosos utilizando desta técnica (COSTA et al., 2016). A própria história oral, usa de métodos semelhantes às contações de estórias, mas com finalidades diferentes (CROOK et al., 1992; SANTOS et al., 2012; YASSUDA et al., 2006). Os estudos que se utilizam de narrativas, de certa forma, usam a estória contada pelos pesquisados como fonte de informação para a análise dos dados. A partir das narrativas individuais é possível utilizar técnicas de análise do discurso ou de conteúdo para apresentação dos resultados. (FERIANI, 2017; MORSCH; MYSKIW, M.; MYSKIW, J., 2016; SANTOS; SANTANA, 2016).

As narrativas sejam elas digitais ou não, são meios que as pessoas têm de deixar um legado de suas memórias para suas famílias, comunidades, ou meios sociais no qual estão inseridas (MCLELLAN, 2007). A possibilidade de fazer isso digitalmente, introduz uma riqueza de informação visual e auditiva, formas de explorar materiais, objetos e fatos de uma forma mais dinâmica e acessível (CUEVA et al., 2013; LETHERBY; DAVIDSON, 2015; MCWILLIAM; BICKLE, 2017).

Desenvolver um projeto de ND, com pessoas idosas, possibilita, não somente, que essas pessoas deixem um legado de suas histórias, bem como, aprendam algo novo, já que em geral as pessoas idosas do presente século, não cresceram tão envolvidas com tecnologia. Aprender algo, por mais simples que seja, possibilita ao indivíduo maior concentração, estimulação do sistema nervoso central e neuroplasticidade sináptica (CECATTO; CHADI, 2012).

$\mathrm{O}$ enorme potencial que as novas tecnologias podem proporcionar na qualidade de vida das pessoas em geral, não passou despercebida para aqueles que trabalham no campo da educação, particularmente, aqueles que trabalham com idosos. O avanço nas tecnologias hoje, permite ao indivíduos, independente 
da idade ou limitações, que possam vir a ter os mesmos direitos e oportunidades de integração social. É importante que toda essa tecnologia não distancie, ainda mais, os idosos da população em geral (DÍAZ-LÓPEZ et al., 2016). Educação e treinamento deve ajudar os idosos a entenderem e utilizarem as novas tecnologias (HUBER; WATSON, 2013).

Pessoas idosas muitas vezes podem apresentar dificuldades com a memória, conforme a idade vai avançando. Possibilitar atividades que estimulem a cognição é muito importante para otimizar esse processo CROOK; FEHER; LARRABEE, 1992; MATTOS et al., 2003).

O uso do computador ocasiona mudanças relacionadas à idade, como a melhora de habilidades psicomotoras e perceptivas (CHIU et al., 2016; OWNBY, 2006), como mudanças na visão, audição, memória percebida, e memória de trabalho. Portanto, aprender a usar um computador e outras tecnologias, possibilita minimizar perdas naturais relativas ao processo de envelhecimento (DÍAZ-LÓPEZ et al., 2016; HUBER; WATSON, 2013).

Alguns estudos que utilizaram a metodologia de ND, têm mostrado que contar estórias, traz um benefício ao resgaste da memória. Quando nessa atividade, o idoso precisa também aprender a utilizar uma tecnologia para gravar essas narrativas e produzir um material áudio-visual, é ainda mais relevante e traz resultados para a recuperação ou manutenção dos processos de memória (HACK et al., 2015; LEE et al., 2014).

\section{Digital Storytelling}

O termo Digital Storytelling, amplamente utilizado na literatura internacional, refere-se à uma atividade muita antiga, contação de histórias, utilizando recursos digitais. No Brasil, o termo mais usado com esse significado tem sido ND (PALÁCIO; STRUCHINER, 2017; RODRIGUES; ALMEIDA; VALENTE, 2017; SANTOS; PORTO JR., 2017).

A técnica de contação de histórias, tem sido trabalhada e conceituada por vários autores nacionais e internacionais, e tem como objetivo levar ao público ouvinte à uma jornada para locais e situações imaginárias, porque mesmo que seja uma história real, o cenário se desenvolverá na mente do ouvinte, a partir de suas próprias memórias sobre a narrativa que está sendo realizada (CUEVA. et al., 2013; MILLER, 2008; PINA, 2009; SOARES; SILVA, 2017; VENDRAME et al., 2017).

A ND irá alcançar sua audiência através de recursos tecnológicos digitais, pois as histórias podem ser disponiblizadas pelos seus criadores em plataformas de vídeo e nas redes sociais. Para produzir essas narrativas são utilizadas ferramentas tecnológicas diversificadas como computadores, celulares inteligentes, filmadoras, recursos da internet, softwares para criação dos vídeos, entre outros recursos.

Algumas características especiais das MD são (MILLER, 2008, p. 17 e 18): eventos dramáticos conectados entre si para contar as estórias; contém caracteres encontrados na mídia digital; é 
interativa, pois os contadores controlam todos os aspectos que a história vai ter; é possível imergir na estória; o criador também participa podendo narrar a estória; pode ser multisensorial; pode incorporar inteligência artificial; os usuários podem criar e controlar avatares; possibilita uma interação entre os membros de uma comunidade; oferece a oportunidade de discussão e mudanças de pontos de vista; pode incluir elementos de fantasia e de jogos.

Miller (2008) também apresenta as importantes diferenças entre a forma clássica e a digital de contar histórias:

Figura 1: Diferenças entre a forma clássica e digital de contar estórias

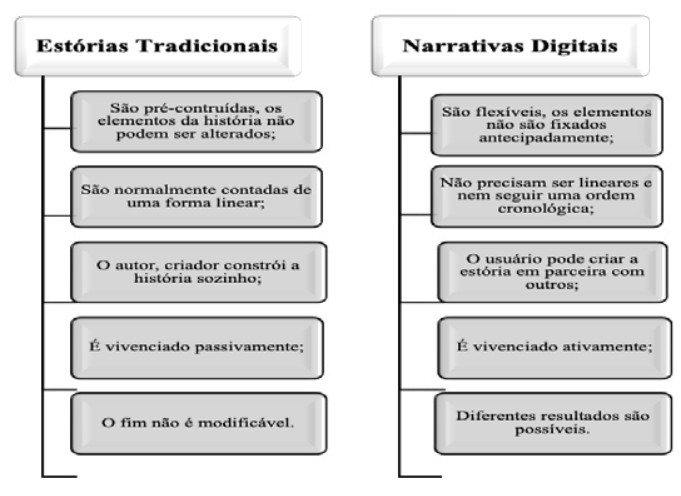

Fonte: Adaptado de Miller (2008, p.19).

A ND tem múltiplos objetivos: a) capacitar os participantes a usarem uma nova tecnologia, possibilitando perceber seu desenvolvimento e crescimento pessoal; b) conscientizar o público que assiste sobre questões apresentadas na história que merecem reflexão; c) informar sobre fatos políticos e históricos; d) fornecer dados narrativos e multisensoriais; e e) apoiar pesquisas e avaliações de saúde pública (GUBRIUM; HILL; FLICKER, 2014).

Em projetos de ND, os participantes normalmente criarão uma narrativa curta e pessoal que pode incorporar vários modos de comunicação, como fotos, gráficos, texto escrito, música, efeitos sonoros, pequenos vídeos, entre outros recursos. $\mathrm{O}$ produto final poderá ser visto em um computador ou outro dispositivo digital. Ao proporcionar múltiplas ferramentas criativas para a expressão desse participante, um projeto de ND oferece novas formas de dar sentido à experiências pessoais, especialmente para adultos e idosos que tiveram pouco acesso aos estudos e ao conhecimento das tecnologias (PRINS, 2016).

O objetivo desse texto é relatar a experiência vivenciada no Canadá, em um projeto de ND desenvolvido num Centro Comunitário em parceria com uma Universidade Pública, com nove idosos, durante 10 semanas.

\section{Metodologia}

\section{Delineamento do estudo}

Este estudo, tratou-se de uma pesquisa qualitativa, já que na seleção dos participantes para o projeto, a amostragem foi intencional, com um número pequeno de sujeitos e o próprio pesquisador aplicou a pesquisa. O estudo utilizou também uma metodologia de estudo de caso, que "Investiga um fenômeno contemporâneo dentro de seu contexto real" (YIN, 2009, p.18). 
Local do estudo

A Pesquisa foi Realizada no Centro Comunitário Kerrisdale, em Vancouver/ Canadá. O Projeto é da Universidade Simon Fraser, Coordenado pelo Professor Dr. David Kaufman.

\section{Material}

Para execução do projeto foi utilizado para elaboração dos vídeos o programa WEVIDEO ${ }^{\circ}$, que possibilita a gravação de áudios, inserção de músicas, fotos, imagens e efeitos especiais. Para a gravação dos áudios, embora possa, ser feitos pelo WEVIDEO $®$, foi utilizado um programa gratuito chamado Audacity ${ }^{\circledR}$, e estes áudios depois foram inseridos no WEVIDEO ${ }^{\circ}$. Nos encontros prévios para ensinar como construir uma boa história, fazer um storyboard e usar o programa WEVIDEO $®$, foi utilizado pelo docente um computador e um projetor. Cada participante também usava um laptop pessoal, ou um emprestado pela universidade.

O projeto possui sempre um facilitador e um co-facilitador. Normalmente $o$ facilitador é um aluno de mestrado, doutorado ou pós-doutorado. O Co-facilitador, pode ser um aluno de graduação, um professor visitante, ou mesmo os alunos de mestrado e doutorado também. Neste projeto, havia um facilitador que era uma aluna de doutorado e uma professora visitante, que foi a co-facilitadora.
O estudo foi conduzido num centro comunitário na cidade de Vancouver, no Estado de British Columbia no Canadá, nos meses de abril e maio de 2017. Participaram deste projeto de ND, nove idosos, sendo 2 homens e 7 mulheres. A idade média do grupo foi de 72 anos, sendo que a mais nova era uma mulher de 66 anos e a mais idosa, tinha 92 anos. $\mathrm{O}$ projeto foi oferecido ao centro comunitário, que divulgou entre os idosos da comunidade, e estes por sua vez se inscreveram. $\mathrm{O}$ projeto teve duração de 10 semanas, com duas horas de duração cada encontro, sempre às quartas-feiras às $10: 00 \mathrm{~h}$. $\mathrm{O}$ projeto não teve custo algum para os participantes, mas solicitou-se que os mesmos trouxessem para as aulas seus computadores (laptops). Para os idosos que não possuíam computador próprio, a Universidade que conduziu o projeto, emprestou os aparelhos durante as 10 semanas do projeto.

A figura 2, ilustra como o projeto ocorreu, durante as 10 semanas. 
Figura 2: Esquema do projeto e os papéis do facilitador e do participante a cada semana

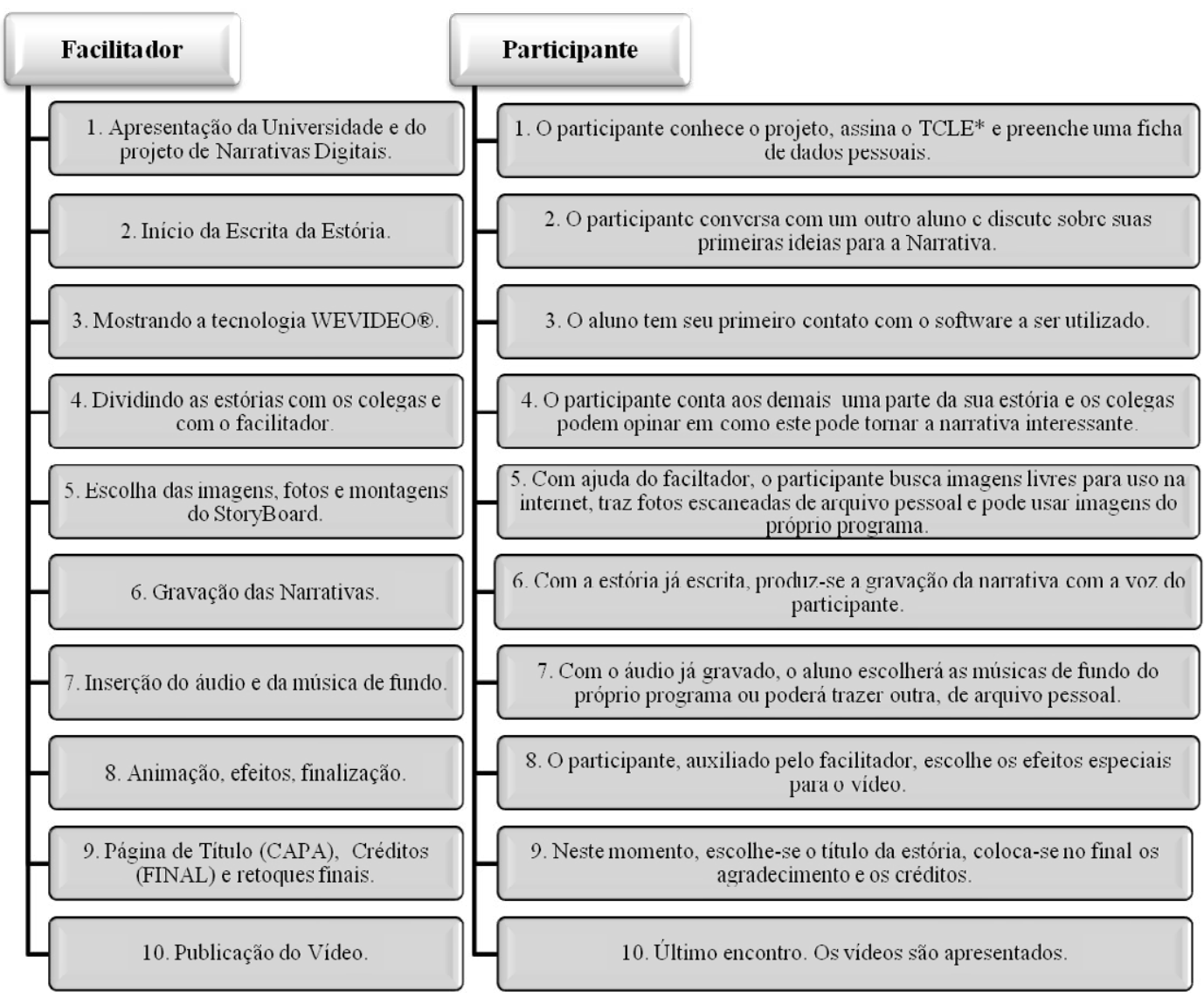

Fonte: Autor (2018)

\section{Relato da experiência}

O primeiro encontro do projeto, é um dia de reconhecimento, apresentação das pessoas, inclusive dos coordenadores do projeto. Nesse dia, é apresentado como o projeto irá funcionar, seus objetivos, a importância de não faltarem e os resultados possíveis. Como o Canadá é um país multicultural, os idosos eram de diferentes nacionalidades. Havia no pro- jeto italianos, ingleses, porto riquenhos, japoneses, chineses, alemães, estadounidenses e canadenses.

Num primeiro momento foi feito um desafio aos participantes, de se apresentarem ao colega que estava ao lado, contando de onde eram, o que gostavam de fazer, seus hobbies, algo a respeito da família e amigos.

Nos encontros seguintes, seguiu-se a sequência metodológica apresentada na 
figura 2. Foi muito interessante observar ao longo dos encontros alguns fatos: a alegria dos participantes em conhecer novas pessoas - alguns moravam no mesmo prédio de apartamento e nunca tinham visto uns aos outros; a satisfação pessoal de aprender a lidar com um programa de computador; a emoção de construir um documento, neste caso, o vídeo, para deixar como legado para os filhos e netos; o carinho manifestado na construção das histórias; o empenho em aprender tudo o que foi necessário para construir o vídeo - escanear fotos, coletar imagens da internet, escolher músicas; e a cortesia com que tratavam cada participante do projeto - muitas vezes os que tiveram maior facilidade auxiliaram os que tiveram mais dificuldade - e os alunos da Universidade.

Nos primeiros encontros o sentimento principal era a preocupação de não conseguir executar a tarefa proposta, o que ao longo das semanas foi sendo dissipado com a alegria de ver pouco a pouco a tarefa sendo bem executada. Ao visualizar vídeos de outros colegas, de projetos anteriores, os participantes tinham receio de não conseguirem fazer vídeos na mesma qualidade e que despertasse interesse no público que os assistiria.

Devido a isso, provavelmente a emoção do último encontro tenha sido tão intensa, pois cada um pode conhecer um pedacinho da história de vida dos participantes. Relembrar histórias do passado, provocou muitas emoções, pois, normalmente, em projetos dessa natureza, as pessoas retratam histórias que marcaram suas vidas, sendo elas positivas ou não.

A satisfação desse grupo de idosos, frequentadores desse centro comunitário, ao participar desse projeto de ND ficou evidente, e alguns queriam saber quando haveria outro projeto desse, para que pudessem participar novamente.

\section{Discussão}

A narração de histórias utilizando a tecnologia é uma forma de transferir conhecimento entre pessoas em diferentes culturas e permite que as pessoas expressem histórias usando imagens, música, sua própria voz e vídeos. As vantagens das ND derivam do aprimoramento de velhos estilos de narração, agora com a utilização de tecnologias digitais. Os alunos idosos reúnem informações, experiências e sentimentos com a produção de conhecimento e apresentam os resultados à família, à comunidade e à sociedade através de recursos digitais (KOCAMAN-KAROGLU, 2016).

Usar recursos semióticos - especialmente os visuais - torna um projeto de ND bastante atraente para a população idosa (PRINS, 2016). As histórias digitais podem oferecer oportunidades de desenvolvimento cognitivo, através da escrita, narração e montagem dos vídeos. Esse projeto, desenvolvido num centro comunitário no Canadá, teve ainda uma característica interessante. Como tratou-se um grupo multicultural, havia vários deles que não eram nativos de um país de língua inglesa, e não tinham a mesma facilidade para escrever e narrar sua his- 
tória em inglês. Tínhamos também uma idosa, que tinha um problema de dicção, mas, tanto ela como os demais, encorajados pelos facilitadores e pelos colegas, executaram as atividades por completo que culminou em vídeos emocionantes.

$\mathrm{O}$ uso de tecnologias digitais pode tornar mais acessível a pesquisa com diferentes populações, como a idosa, que é o caso deste relato. Os pesquisadores podem fazer uso desse tipo de workshop de ND, como um espaço para a realização de pesquisa etnográficas, incorporando observações participantes e notas nos diários de campo, realizando entrevstas individuais e grupais (GUBRIUM; HILL; FLICKER, 2014).

Embora exista um crescente números de estudos e projetos sobre ND, poucos estudos têm explorado essa técnica com a população idosa, avaliando algumas características do processo de envelhecimento, como metamemória, cognição, entre outros fatores que se modificam ao longo da vida. Além disso, para o idoso participar de um projeto como esse, possibilita que este possa "deixar um legado", para que outros saibam mais sobre suas vidas aprendendo com suas experiências (MANCHESTER; FACER, 2015).

\section{Considerações Finais}

Empiricamente, as descobertas do estudo contribuem para o campo de ensino de pessoas idosas ao mostrar como a edição de um vídeo por meio de ferramentas tecnológicas podem promover a competência digital de pessoas idosas, que usualmente tem pouco acesso às ferramentas digitais, mesmo em países desenvolvidos como o Canadá, além de entrarem em contato com a tecnologia tardiamente. Como contribuição teórica, o estudo mostrou um modelo de aplicação de um workshop de ND, que pode ser usado em diferentes populações e não somente com as pessoas idosas.

Este projeto que foi desenvolvido com idosos de um centro comunitário no Canadá, pode inspirar educadores para incorporarem as ND no processo de ensino e aprendizagem de pessoas idosas.

\section{Storytelling digital project: working digital narratives with older adults}

\section{Abstract}

The objective of this research is to report the experience in the participation of a Digital Storytelling Project which provides the elaboration of stories by the participants. This project occurred in 10 weeks, in a workshop format, where the researcher teaches the participants how to write a story that he/she wants to tell for someone; informed what resources will be used in this process, and what materials will be needed for the participant to create a good story. This work was carried out with elderly people who were invited to participate in that workshop. For this project a software was used to record the voice of the participant; placement of the images and assembly of the sound reconstruction. Studies in Canada with Digital Narratives among the elderly population have shown positive results in social interaction, cognitive stimulation and perceived well-being.

Key-words: Storytelling. Digital. Elderly. Technology. 


\section{Agradecimentos}

À direção do Centro Comunitário Kerrisdale, em Vancouver no Canadá por possibilitar a execução do projeto de Narrativas Digitais com os idosos da comunidade.

Ao professor David Kaufman do departamento de Educação da Simon Fraser University e sua equipe por me convidar para participar desse projeto.

À Simon Fraser University e ao AGE-WELL NCE, por financiar o projeto.

\section{Referências}

CECATTO, R. B.; CHADI, G. Functional electrical stimulation (FES) and neuronal plasticity: a historical review. Acta Fisiátrica, v. 19 , n. 4, p. 246-257, 2013. DOI: 10.5935/0104-7795.20120040.

CHIU, C. J. et al. The attitudes, impact, and learning needs of older adults using apps on touchscreen mobile devices: Results from a pilot study. Computers in Human Behavior, v. 63 , p. 189-197, 2016. DOI: https:// doi.org/10.1016/j.chb.2016.05.020.

COSTA, N. P. DA et al. Contação de história: tecnologia cuidativa na educação permanente para o envelhecimento ativo. Revista Brasileira de Enfermagem, v. 69 , n. 6, p. 1132-1139, 2016. DOI: https://doi. org/10.1590/0034-7167-2016-0390.

CROOK III, T. H.; FEHER, E. P.; LARRABEE, G. J. Assessment of memory complaint in age-associated memory impairment: the MAC-Q. International psychogeriatrics, v. 4 , n. 2 , p. $165-176$, 1992. DOI: $10.1017 /$ s1041610292000991.

CUEVA, M. et al. Bridging storytelling traditions with digital technology. International
Journal of Circumpolar Health, v. 72, n. SUPPL.1, p. 1-6, 2013. DOI: 10.3402/ijch. v72i0.20717.

DÍAZ-LÓPEZ, DEL M. P. et al. Keys to active ageing: new communication technologies and lifelong learning. SpringerPlus, v. 5, n. 1, p. 1-8, 2016. DOI: 10.1186/s40064-016-2434-8.

FERIANI, D. Demência(s), em imagens, narrativas e experiências. Revista Brasileira de Ciências Sociais, v. 31, n. 93, p. 1-5, 2017. DOI: https://doi.org/10.17666/329314/2017.

GUBRIUM, A. C.; HILL, A. L.; FLICKER, S. A situated practice of ethics for participatory visual and digital methods in public health research and practice: A focus on digital storytelling. American Journal of Public Health, v. 104, n. 9, p. 1606-1614, 2014. DOI: 10.2105/AJPH.2013.301310.

HACK, J. R. et al. Lifelong learning through digital storytelling in corporate training Aprendizaje permanente basado en el uso de las historias digitales en la formación empresarial. Revista Complutense de Educación, v. 26 , n. 2, p. 351-365, 2015. DOI: 10.5209/REV_RCED.2015.V26.N2.43109.

HUBER, L.; WATSON, C. Technology: Education and Training Needs of Older Adults. Educational Gerontology, v. 40, n. 1, p. 16-25, 2013. DOI: https://doi.org/10.1080/0 3601277.2013.768064.

KOCAMAN-KAROGLU, A. Personal voices in higher education: A digital storytelling experience for pre-service teachers. Education and Information Technologies, v. 21, n. 5 , p. $1153-1168,2016$. DOI: http://dx.doi. org/10.1007/s10639-014-9373-1.

KOTLUK, N. Researching and Evaluating Digital Storytelling As a Distance Education Tool in Physics Instruction : an Application With Pre-Service Physics Teachers. Turkish Online Journal of Distance Education (TOJDE), v. 17, n. 1, p. 87-99, 2015. DOI: 10.17718/tojde.59900.

LEE, H.-C. et al. Picgo: Designing Reminiscence and Storytelling for the Elderly with 
Photo Annotation. Designing Interactive Systems, v. 21, n. jun, p. 9-12, 2014. DOI: 10.1145/2598784.2602769.

LETHERBY, G.; DAVIDSON, D. Embodied Storytelling: Loss and Bereavement, Creative Practices, and Support. Illness, Crisis \& Loss, v. 23, n. 4, p. 343-360, 2015. DOI: https://doi.org/10.1177/1054137315590745.

MANCHESTER, H.; FACER, K. Digital curation: Learning and legacy in later life. E-Learning and Digital Media, v. 12, n. 2, p. 242-258, 2015. DOI: https://doi. org/10.1177/2042753014568178.

MATTOS, P. et al. Memory complaints and test performance in healthy elderly persons. Arquivos de neuro-psiquiatria, v. 61, n. 4, p. 920-4, 2003. DOI: http://dx.doi. org/10.1590/S0004-282X2003000600006.

MCLELLAN, H. Digital Storytelling in Higher Education. Journal of Computing in Higher Education, v. 19, n. 1, p. 65-79, 2007. DOI: https://doi.org/10.1007/ BF03033420.

MCWILLIAM, K.; BICKLE, S. Digital storytelling and the 'problem' of sentimentality. Media International Australia, v. 165 , n. 1, p. 77-89, 2017. DOI: 10.1177/1329878X17726626.

MILLER, C. H. Digital Storytelling: a creator's guide to interactive entertainment. $2^{\text {nd }}$ ed.. Oxford: ELSEVIER, 2008. DOI: 10.1201/9780429440045.

MORSCH, P.; MYSKIW, M.; MYSKIW, J. DE C. A problematização da queda e a identificação dos fatores de risco na narrativa de idosos. Ciência \& Saúde Coletiva, v. 21, n. 11, p. 3565-3574, 2016. DOI: http://dx.doi. org/10.1590/1413-812320152111.06782016.

OWNBY, R. L. Making the internet a friendlier place for older people. Generations, v. 30, n. 2 , p. 58-60, 2006. Disponível em: https://www.questia.com/library/journal/1P3-1219307591/making-the-internet-a-friendlier-place-for-older-people.
PALÁCiO, M. A. V.; STRUCHINER, M. Análise da produção de narrativas digitais no ensino superior em saúde. EaD em FOCO, v. 7, n. 1, p. $62-71,2017$. DOI: https://doi. org/10.18264/eadf.v7i1.541.

PINA, P. K. D. C. Esboço de uma poética da leitura em Dom Quixote das crianças de Monteiro Lobato. Leitura: Teoria \& Prática, v. 27 , n. 52 , p. 34-40, 2009. DOI: https://doi. org/10.34112/2317-0972a2009v27n52p34-40.

PRINS, E. Digital storytelling in adult education and family literacy: a case study from rural Ireland. Learning, Media and Technology, v. 42, n. 3, p. 1-16, 2016. DOI: 10.1080/17439884.2016.1154075.

RODRIGUES, A.; DE ALMEIDA, M. E. B.; VALENTE, J. A. Currículo, narrativas

digitais e formação de professores: Experiências da pós-graduação à escola. Revista Portuguesa de Educação, v. 30, n. 1, p. 61, 2017. DOI: $10.21814 /$ rpe.8871.

SANTOS, A. T. DOS et al. Queixa subjetiva de comprometimento da memória em idosos saudáveis: influência de sintomas depressivos, percepção de estresse e autoestima. Revista da Escola de Enfermagem da USP, v. 46 , n. spe, p. 24-29, 2012. DOI: https:// doi.org/10.1590/S0080-62342012000700004.

SANTOS, K. P. DOS; SANTANA, A. P. A linguagem dos idosos da região sudeste do Brasil: o nível prosódico. Acta Scientiarum. Language and Culture, v. 38, n. 4, p. 371, 2016. DOI: https://doi.org/10.4025/actascilangcult.v38i4.27687.

SANTOS, M. C. DOS; PORTO JR, F. G. R. Tecnologia e narrativas digitais digitais : por entre caminhos. Revista Observatório, v. 3, n. 3, p. 24-33, 2017. DOI: https://doi. org/10.20873/uft.2447-4266.2017v3n3p24.

SOARES, A. R.; SILVA, G. M. D. D. Contação de história: um olhar a partir da ludicidade. XXIII SEMINÁRIO DE INICIAÇÃO CIENTÍFICA - SIEPE 2017, v. 1, n. 1, p. 1, 2017. Disponível em: https://portalperiodicos. unoesc.edu.br/siepe/article/view/14603/7712. 
VENDRAME, E. C. D. S. et al. Era uma vez... a contação de histórias como ação humanizadora no hemocentro. Cadernos de pesquisa: pensamento educacional, v. 12 , n. 30 , p. 176-190, 2017. DOI: 10.35168/2175-2613. UTP.pens_ed.2017.Vol12.N30.pp176-190.

YASSUDA, M. et al. Treino de memória no idoso saudável: benefícios e mecanismos. Psicologia: reflexão e critica, v. 19, n. 3, p. 470-481, 2006. DOI: https://doi.org/10.1590/ S0102-79722006000300016.

YIN, R. K. Case Study Research: Design and Methods (4 ed.). Thousand Oaks, Calif.: Sage, 2009. DOI: $10.2307 / 41262395$. 